\title{
A Novel Sandwich-type Traveling Wave Pi- ezoelectric Tracked Mobile System
}

Liang Wang ${ }^{\mathrm{a},{ }^{*}}$, Chengyou Shu ${ }^{\mathrm{a}}$, Quan Zhang ${ }^{\mathrm{b}}$, Jiamei Jin ${ }^{\mathrm{a}}$ 


\begin{abstract}
:
In this paper, a novel sandwich-type traveling wave piezoelectric tracked mobile system was proposed, designed, fabricated and experimentally investigated. The proposed system exhibits the advantages of simple structure, high mechanical integration, lack of electromagnetic interference, and lack of lubrication requirement, and hence shows potential application to robotic rovers for planetary exploration. The tracked mobile system is comprised of a sandwich actuating mechanism and a metal track. The actuating mechanism includes a sandwich piezoelectric transducer and two annular parts symmetrically placed at either end of the transducer, while the metal track is tensioned along the outer surfaces of the annular parts. Traveling waves with the same rotational direction are generated in the two annular parts, producing the microscopic elliptical motions of the surface particles on the annular parts. In this situation, if the pre-load is applied properly, the metal track can be driven by friction force to achieve bidirectional movement. At first, the finite element method was adopted to conduct the modal analysis and harmonic response analysis of the actuating mechanism, and the vibration characteristics were measured to confirm the operating principle. Then the optimal driving frequency of the system prototype, namely $35.1 \mathrm{kHz}$, was measured by frequency sensitivity experiments. At last, the mechanical motion characteristics of the prototype were investigated experimentally. The results show that the average motion speeds of the prototype in dual directions were as $72 \mathrm{~mm} / \mathrm{s}$ and $61.5 \mathrm{~mm} / \mathrm{s}$ under the excitation voltage of $500 \mathrm{~V}_{\mathrm{RMS}}$, respectively. The optimal loading weights of the prototype in bi-directions were $0.32 \mathrm{~kg}$ and $0.24 \mathrm{~kg}$ with a maximum speed of $59.5 \mathrm{~mm} / \mathrm{s}$ and $61.67 \mathrm{~mm} / \mathrm{s}$ at the driving voltage of $300 \mathrm{~V}_{\mathrm{RMS}}$, respectively.
\end{abstract}

Keywords: Tracked mobile system; sandwich piezoelectric transducer; actuating mechanism; metal track; experimental investigations. 


\section{Introduction}

Planetary exploration, which has been given high priority and heavy financial investment by most of the world's countries, is an important aspect of space science [1]-[3]. Robotic rovers act as actuators and carriers of scientific instruments for planetary exploration missions [4]. Their carrying capacity, mobility, and environmental adaptability are benchmarks of quality that ensure overall efficiency and reliable performance [5].

Tracked mechanisms can offer low mean maximum pressure during the driving process [6], [7], in addition to low vehicle sinkage, low mobile resistance when moving through soft soil [8], and large traction force. As such, this technology shows attractive potential in terms of planetary exploration applications. The tracked mechanism increases the contact area between the machine and the ground as the tracks follow the contours of the terrain, almost invariably creating high drawbar pull when moving across rough ground. To this effect, tracked robots are highly mobile across, say, an uneven planet surface [9]-[12].

Traditional tracked mobile robots are generally comprised of a tread, a drive sprocket, an idler-wheel (or a powered wheel,) and an optional number of supporting bogie wheels, creating relatively complex gear trains [10]-[14]. There are noticeable drawbacks to this type of machine, including high power consumption, low operation efficiency, and the necessity for complex control systems [15]. Researchers and developers have attempted to mitigate these drawbacks by designing miniature tracked mobile robotic rovers for use in off-road applications [16]-[19]. The European Space Agency, for example, developed a miniature rover called 'Nanokhod' for employ in planetary exploration; Nanokhod was able to climb obstacles up to $0.1 \mathrm{~m}$ in height and at a maximum speed of $5 \mathrm{~m} / \mathrm{h}$, but its tracked miniature rover mechanism was still too complex to be practical and high internal friction weakens its mobility [17].

In effort to improve upon the traditional tracked robots while retaining its original advantages and, at the same time, to integrate the merits of compact structure, large force (torque) weight ratio, environmental adaptability, and structural diversification of the piezoelectric ultrasonic motors [20]-[26], a novel miniature tracked vehicle driven by a pair of bonded-type parallelogram-frame-structure piezoelectric transducer was developed in our previous work [27]. The miniature piezoelectric tracked vehicle eliminates the complex driving mechanisms of traditional tracked robots, instead using one pair of piezoelectric transducers to actuate the tracks. This allows for simple structure without sliders or bearings, bogie wheels, or track links, and importantly, without lubricants, which thoroughly eliminates the problems of lubricant volatilization and deflation when operating in vacuum environment. Experimental results showed that the vehicle can reach a limited velocity of $4.3 \mathrm{~mm} / \mathrm{s}$ under an excited voltage of $250 \mathrm{~V}_{\mathrm{RMS}}$.

The miniature piezoelectric-actuated tracked vehicle represents a novel and quite promising approach to tracked robotic rovers designed for planetary exploration. However, several issues require attention before the tracked vehicle can be employed in practice. For one, the vehicle has low motion 
speed on account of the piezoelectric ceramics (Lead zirconate titanate, PZT) glued onto the parallelogram frame surfaces to excite the required vibration modes of the piezoelectric transducer; the $\mathrm{d} 31$ working mode of PZT, in effect, is a problem with week stimulating effect. In addition, the PZTs are easily cracked during the vibration procedure due to the limited strength and fatigue life of the glue layer, leaving the entire tracked vehicle relatively unstable. Further, the plastic track used in the study mentioned above was a flawed design choice, because most of the micro amplitude vibration of the piezoelectric transducer was absorbed and dissipated instead of actuating the track.

In effort to improve upon previous piezoelectric tracked vehicle design, we propose a traveling wave piezoelectric tracked mobile system driven by a novel sandwich actuating mechanism utilizing the d33 piezoelectric ceramic vibration mode and friction effect. The proposed system showed advantages of higher electromechanical coupling efficiency of energy conversion compared to the d31 vibration mode, large driving force, and compact structure. It also possesses all merits of the previous piezoelectric tracked vehicle [27], such as functionality without lubricant and lack of electromagnetic interference. In addition, the sandwich actuating mechanism vibrates at the ultrasonic frequency range, effectively producing self-cleaning functions and avoiding dust invasion in contact interfaces. We took the metal track from a toy tank, reprocessed it, and used it to assemble a sandwich actuating mechanism to structure our piezoelectric tracked mobile system, which we observed to significantly enhance the driving efficiency of the actuating mechanism. The proposed sandwich-type traveling wave piezoelectric tracked mobile system likely shows potential application to robotic rover technologies.

The reminder of this paper is organized as follows. At first, the structure design and operating principle of the proposed system are presented and explained in detail, and then the modal analysis and harmonic response analysis of the sandwich actuating mechanism are calculated via the finite element method (FEM). Finally, experimental investigations are carried out to reveal the inherent mechanical properties of the sandwich-type traveling wave piezoelectric tracked mobile system.

\section{Configuration and principle}

\subsection{Structure design}

By exploiting the merits of standing wave Langevin transducers [21], such as high power density and high reliability, a novel traveling wave sandwich actuating mechanism with dual rings is proposed to create a track driver. It is comprised of two annular parts and a variable cross-section sandwich piezoelectric transducer including a pair of variable cross-section beams, a connection block, four sets of PZTs and four steel bolts. As mentioned above, the two annular parts are symmetrically placed at both ends of the variable cross-section sandwich piezoelectric transducer, and four sets of PZTs are located at the terminal vertexes of the connection block and clamped between the connection block and two rectangular variable cross-section beams with steel bolts. The actuating mechanism is, as such, a symmetrical structure with the connection block as its axis of symmetry, as shown in Fig. 1(a). To adjust proper preload forces for PZTs on bolts during assembly of the actuating mechanism, the connection 
block material must have high strength to prevent bolt loosening due to the PZT ultrasonic vibration.

We designed the beams in a stepped shape to magnify the vibration amplitudes of the annular parts. Two rectangular piezoelectric elements with opposite polarization directions comprise a set of PZTs, all of which are polarized along their thickness directions (OX direction) as shown in Fig. 1(a). To achieve optimal actuating effect, a metal track (taken from a toy tank,) made of Zn-Fe alloy was tensioned along the outer surface of the annular parts of the actuating mechanism to constitute the sandwich-type traveling wave piezoelectric tracked mobile system shown in Fig. 1(b). It was employed to satisfy driving requirements of flexibility, rigidity, and roughness of contact interface; the requirements were derived from our previous research on contact and friction behavior experimental results of the bonded-type piezoelectric transducer [28], as also shown in Fig. 1(b). Flexibility is the critical factor in realizing the continuous movement of a track driven by the actuating mechanism, so we connected a number of track blocks with metal pins to constitute the metal track to keep it highly flexible; the track also was highly rigid due to the $\mathrm{Zn}-\mathrm{Fe}$ alloy material. The area of the track that contacts the annular parts of the actuating mechanism was processed until entirely smooth to ensure that the roughness of the contact interface matched that of the track material. The pre-stress between the metal track and actuating mechanism was determined by the length of the track, directly influencing the driving performance of the system.

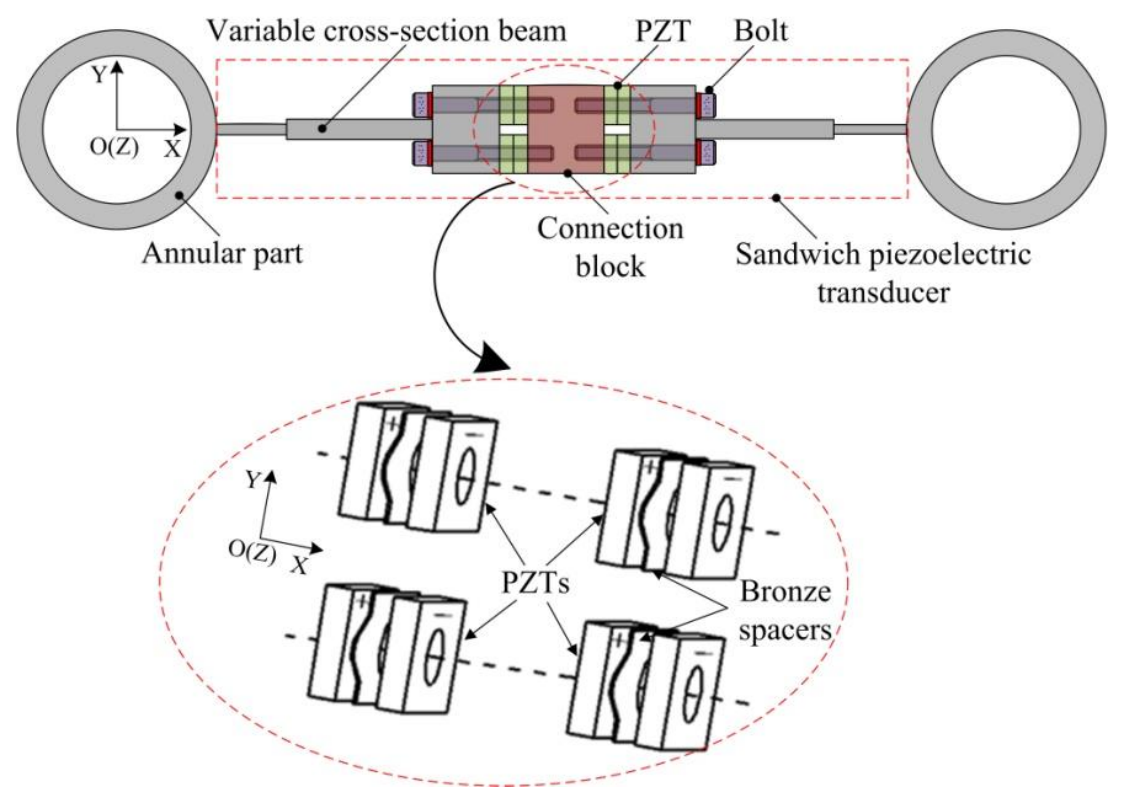

(a) 


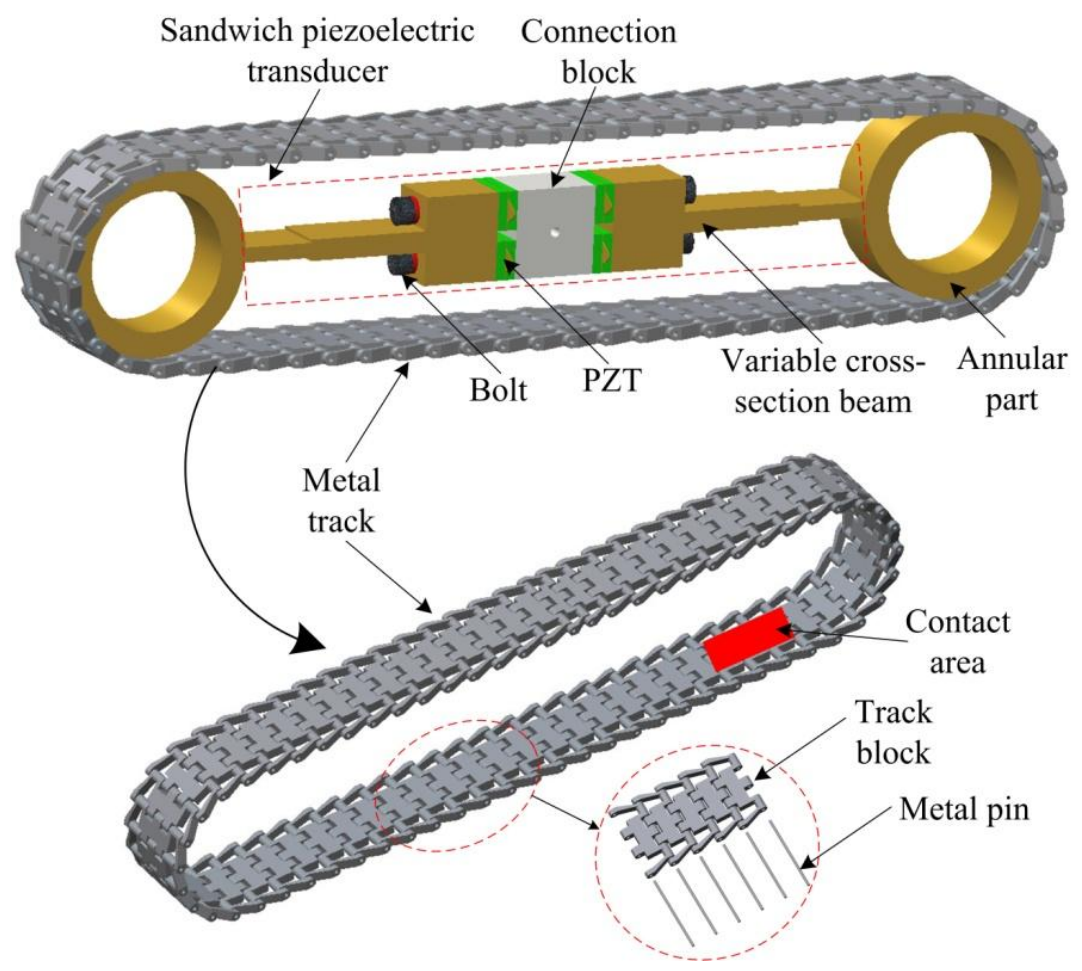

(b)

Fig. 1. Configuration view of the sandwich-type traveling wave piezoelectric tracked mobile system and details. (a) Configuration of the proposed sandwich actuating mechanism and the polarization of piezoelectric ceramics. (b) Sketch of the sandwich-type traveling wave piezoelectric tacked mobile system and detailed structure of the metal track.

\subsection{Operating principle}

To drive the metal track, traveling wave with the same rotating direction must be produced in the two annular parts of the sandwich-type actuating mechanism; thus, the generation of traveling waves is the key step in designing the actuating mechanism successfully. As discussed above, the longitudinal and bending vibration modes of the variable cross-section sandwich piezoelectric transducer were selected as the operating modes to excite traveling waves of the annular parts. As four sets of PZTs were supplied with a sinusoidal signal simultaneously, the variable cross-section sandwich piezoelectric transducer was excited to vibrate at a third-order longitudinal vibration mode $\left(\mathrm{L}_{3}\right)$ and the annular parts exhibited a fourth-order in-plane bending vibration mode $\left(\mathrm{B}_{04}\right)$, as shown in Fig. 2(a). If two sets of PZTs located at one of diagonals were supplied with a cosine signal, the variable cross-section sandwich piezoelectric transducer would vibrate at a tenth-order bending vibration mode $\left(\mathrm{B}_{10}\right)$ and the annular parts in another fourth-order in-plane bending vibration mode $\left(\mathrm{B}_{04}\right)$, as shown in Fig. 2(b). To stimulate the longitudinal and bending vibration modes in the variable cross-section sandwich piezoelectric transducer simultaneously, two phase excitation signals with a temporal phase difference of $\pi / 2$ were applied to the diagonal PZTs, respectively, and thus two in-plane bending vibration modes were generated in the annular parts with a spatial phase difference of $\pi / 2$, as shown in Fig. 2(c). In short, necessary conditions were met for forming traveling waves with the same rotating direction in the annular parts. The surface particles of the annular parts moved in a microscopic elliptical trajectory, while 
the track was driven by friction force, as shown in Fig. 2(d), while a pair of electric signals with $-\pi / 2$ phase shift were applied to the PZTs at the two respective diagonals; the traveling waves then formed in the annular parts reverse rotated to drive the metal track in the opposite direction.

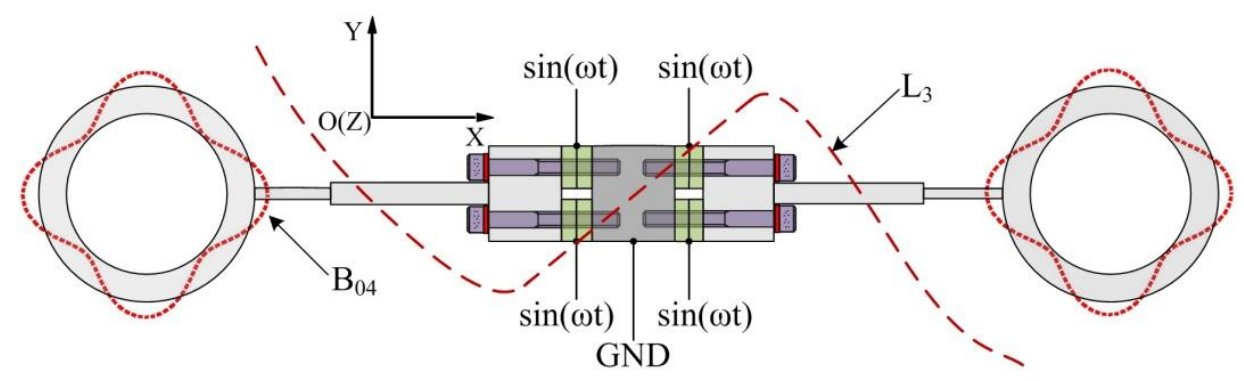

(a)

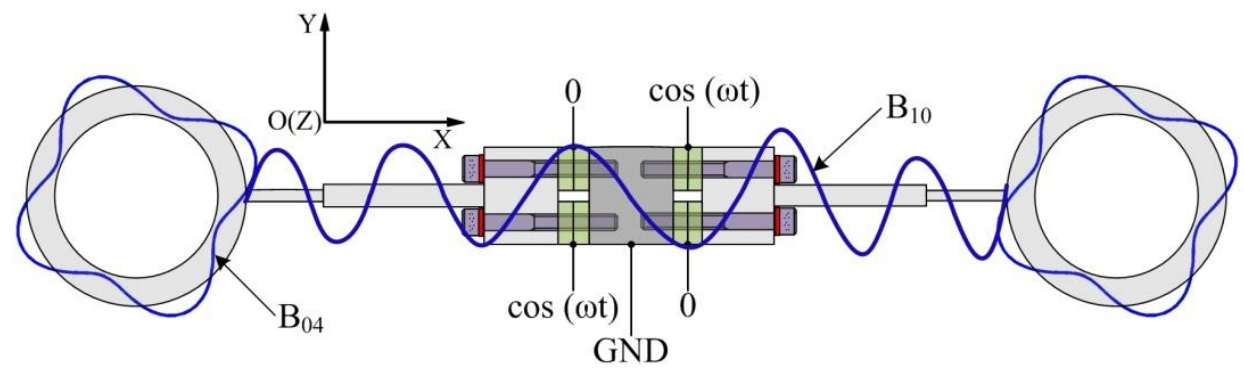

(b)

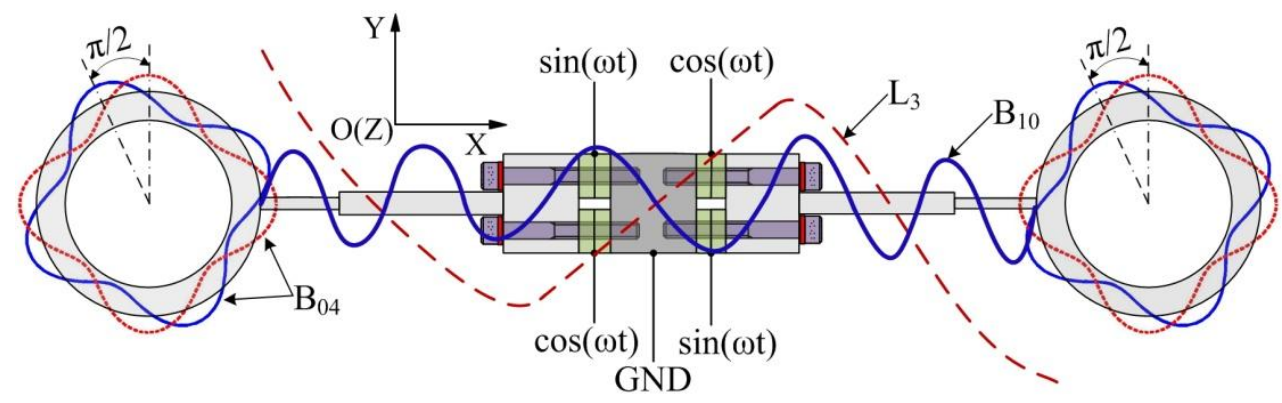

(c)

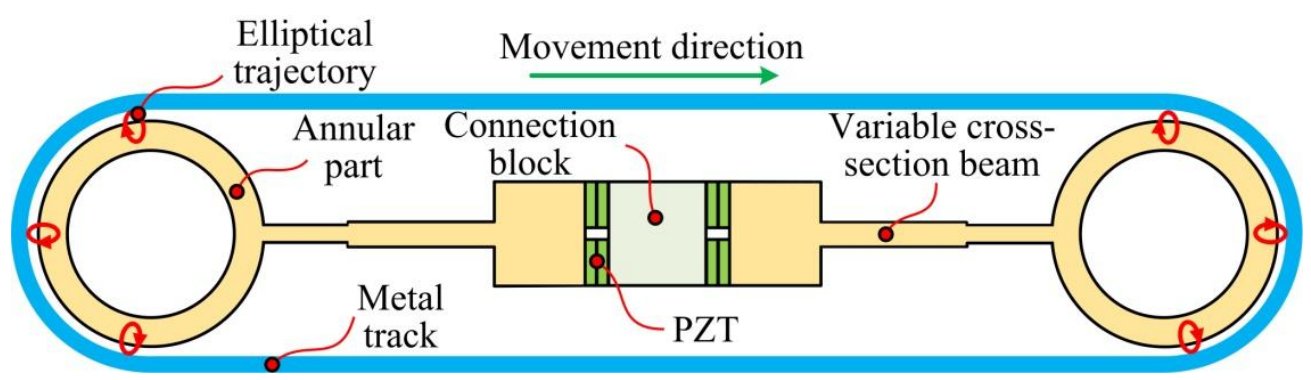

(d)

Fig. 2. Operating principles of the sandwich-type actuating mechanism and tracked mobile system. (a) In-plane $B_{04}$ vibration mode in annular parts with the $\mathrm{L}_{3}$ vibration mode in the sandwich piezoelectric transducer. (b) Another in-plane $\mathrm{B}_{04}$ vibration mode in annular parts with the $\mathrm{B}_{10}$ vibration mode in the sandwich piezoelectric transducer. (c) Coupling of two vibration modes of the sandwich actuating mechanism. (d) Operating principle of the sandwich-type tracked mobile system.

\section{FEM simulation of the actuating mechanism}

\subsection{Modal analysis}


We used commercial ANSYS software to conduct modal analysis. First, we designed the appearance sizes of the actuating mechanism and calculated its vibration modes. A variable cross-section beam and an annular part made of phosphor bronze were combined, and then high-strength 65Mn was used to build the connection block. The piezoelectric elements selected were PZT- $8\left(\mathrm{~d}_{33}=250 \mathrm{pC} / \mathrm{N}, \mathrm{K}_{\mathrm{p}}=0.31\right.$, $\mathrm{Q}_{\mathrm{m}}=1500$ ). All material parameters are listed in table 1. The geometric sizes of the PZTs were $12 \mathrm{~mm} \times 8 \mathrm{~mm} \times 2 \mathrm{~mm}$ with a center hole $4 \mathrm{~mm}$ in diameter, so the width of the initial actuating mechanism model was a fixed constant. The geometric parameters of the actuating mechanism are illustrated in Fig. 3. The unfixed geometric parameters (p1-p9) of the model were set to range within a specific threshold during the modal analysis. After a series of parameters adjustments to filtrate the planning vibration modes, we identified the optimized actuating mechanism model in terms of apparent component sizes, as listed in table 2 .

Table 1 Material parameters of the actuating mechanism.

\begin{tabular}{cccc}
\hline Materials & Young's modulus $(\mathrm{Pa})$ & Poisson's ratio & Density $\left(\mathrm{kg} / \mathrm{m}^{3}\right)$ \\
\hline Phosphor bronze & $1.13 \times 10^{11}$ & 0.33 & $8.8 \times 10^{3}$ \\
$65 \mathrm{Mn}$ & $2.11 \times 10^{11}$ & 0.288 & $7.82 \times 10^{3}$ \\
PZT-8 & 1 & 1 & $7.6 \times 10^{3}$ \\
\hline
\end{tabular}

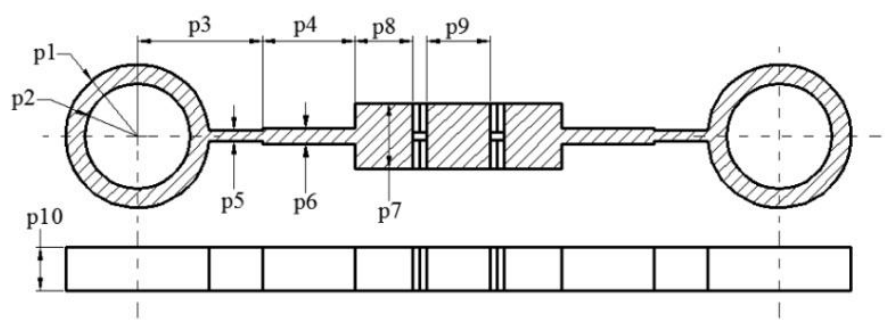

Fig. 3. Geometric parameters of the actuating mechanism.

Table 2 Optimized geometric parameters of the actuating mechanism.

\begin{tabular}{ccccccccccc}
\hline Parameters & p1 & p2 & p3 & p4 & p5 & p6 & p7 & p8 & p9 & p10 \\
\hline Values (mm) & 20 & 15 & 35 & 25 & 2 & 4 & 20 & 15 & 20 & 8
\end{tabular}

The calculated results of the working vibration modes are shown in Fig. 4. When the sandwich piezoelectric transducer vibrated at the $\mathrm{L}_{3}$ vibration mode, the annular parts presented the in-plane $\mathrm{B}_{04}$ vibration mode and the ends of the sandwich piezoelectric transducer were at the crest or trough positions of the in-plane $\mathrm{B}_{04}$ vibration mode simultaneously, as shown in Fig. 4(a). In this case, the eigen-frequency of the actuating mechanism was $36.945 \mathrm{kHz}$. Another in-plane $\mathrm{B}_{04}$ vibration mode appeared in the annular parts when the sandwich piezoelectric transducer exhibited the $\mathrm{B}_{10}$ vibration mode, where the ends of the sandwich piezoelectric transducer were at the nodes of the in-plane $\mathrm{B}_{04}$ vibration mode, as shown in Fig. 4(b). In this case, the resonant frequency was $36.937 \mathrm{kHz}$. The resonant frequency difference between the two working vibration modes was only $8 \mathrm{~Hz}$, indicating that FEM analysis does accurately describe the actuating mechanism. Furthermore, the two bending vibration modes in each annular part had a spatial phase difference of $\pi / 2$, so the calculated results confirm 
that traveling waves successfully formed with the same rotational direction in the annular parts.

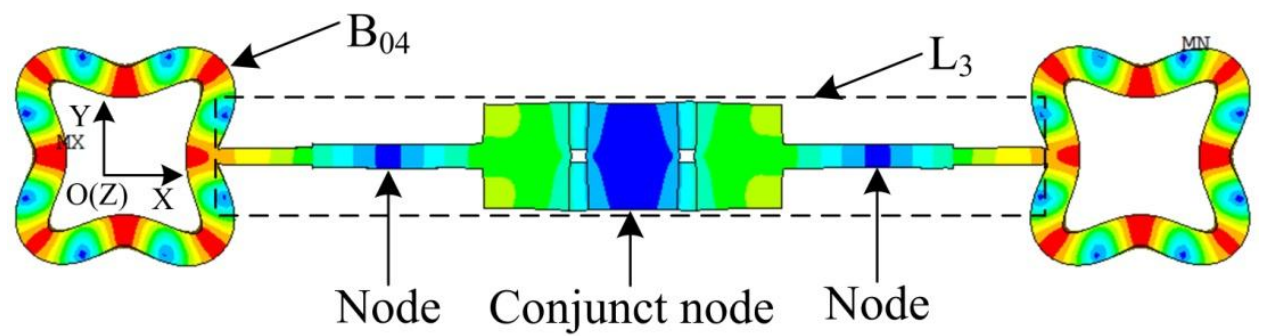

(a)

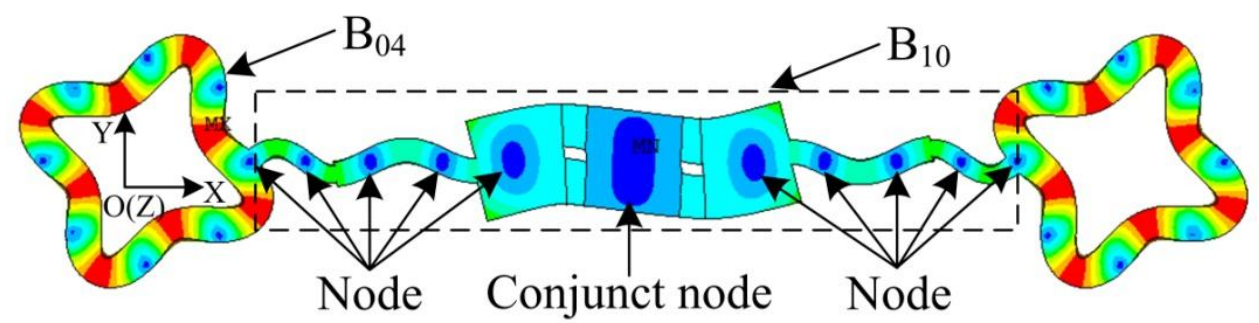

(b)

Fig. 4. Working vibration modes of the sandwich-type actuating mechanism calculated by FEM. (a) In-plane $\mathrm{B}_{04}$ vibration mode presented in annular parts with the $\mathrm{L}_{3}$ vibration mode in the sandwich piezoelectric transducer. (b) Another in-plane $\mathrm{B}_{04}$ vibration mode appeared in annular parts when the sandwich piezoelectric transducer vibrates at the $\mathrm{B}_{10}$ vibration mode.

\subsection{Harmonic response analysis}

The resonant frequencies of the two operating vibration modes must be tuned closely so the traveling waves formed in the annular parts will become "pure" and the elliptical movements of the surface particles on the annular parts will be effectively generated. Therefore, according to the results of modal analysis, harmonic response analysis was conducted using FEM simulation to tune the resonant frequencies of the two operating vibration modes. Under the excitation of two electric signals with a temporal phase difference of $\pi / 2$ (Fig. 5(a)), the optimized operating vibration shape of the actuating mechanism was obtained after tuning the eigen-frequencies, as illustrated in Fig. 5(b). The tuned exciting frequency for the actuating mechanism was $36.94 \mathrm{kHz}$ after harmonic response analysis. One exciting period can be divided into four time stages, as described by different color parts in Fig. 5(a). During one period, the operating vibration shape changed as (1)-(2)-(3)-(4) after applying electric voltages as shown in Fig. 5(a). While the sequence of the electric signal was exchanged, the operating vibration shape changed as (1)-(4)-(3)-(2).

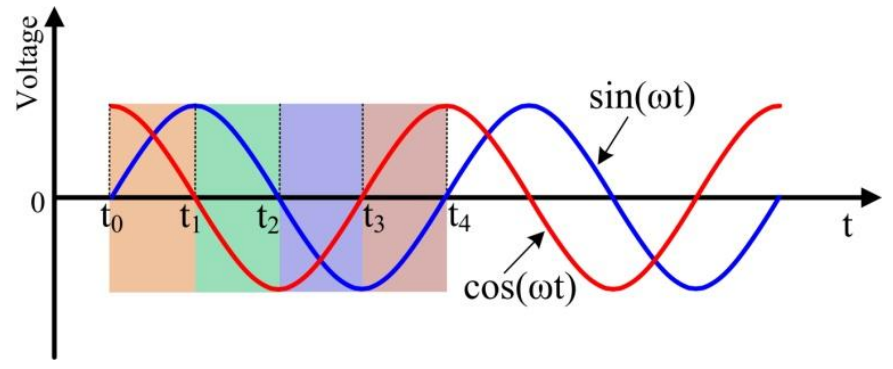


(a)
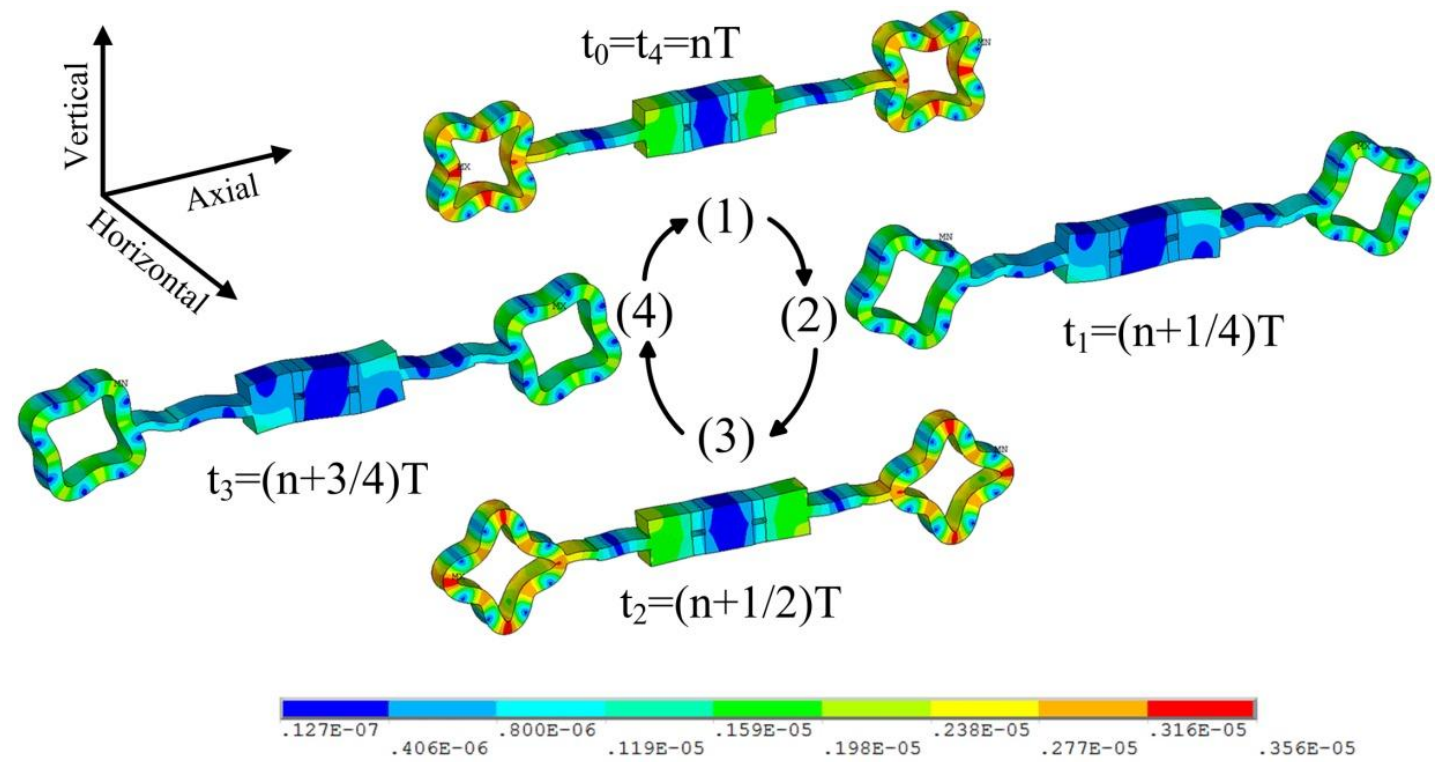

(b)

Fig. 5. Harmonic response analysis results of the actuating mechanism via FEM simulation. (a) Exciting sequence of the electric signals. (b) Operating vibration shapes calculated by FEM simulation during a working period.

\section{Experiments and analysis}

We manufactured a prototype of the sandwich-type piezoelectric tracked mobile system and assembled it based on the above design parameters, as shown in Fig. 6. The weight of the whole system is $0.403 \mathrm{~kg}$.

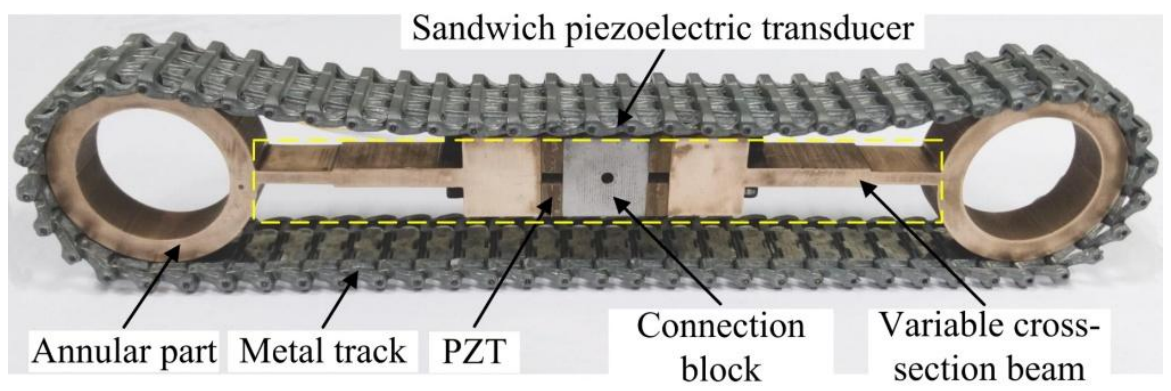

Fig. 6. Prototype of the sandwich-type piezoelectric tracked mobile system.

\subsection{Vibration characteristics}

To effectively excite the traveling waves with the same rotational direction in the two annular parts, we conducted an experiment to tune the resonant frequencies of the two operating vibration modes of the actuating mechanism prototype as closely as possible. The vibration characteristics of the actuating mechanism were measured with a Laser Doppler Vibrometer (Polytec, PSV-300F-B). Because the vibration modes in the curved surfaces of the annular parts could not be measured directly, we measured their terminal faces; due to Poisson effect, the terminal faces of the annular parts exhibit out-of-plane vibration as the actuating mechanism is stimulated to vibrate. The vibration characteristic experiment 
results are illustrated in Fig. 7. When all four sets of PZTs were supplied with an exciting voltage of $100 \mathrm{~V}_{\mathrm{RMS}}$, the measured resonant frequency was $35.335 \mathrm{kHz}$, at which point the sandwich piezoelectric transducer was excited to vibrate at the $\mathrm{L}_{3}$ vibration mode and the annular parts at the in-plane $\mathrm{B}_{04}$ vibration mode, as shown in Fig. 7(a). When two sets of PZTs located at each diagonal were driven with the same voltage and the other sets of PZTs were short circuited, the measured resonant frequency was $35.063 \mathrm{kHz}$ and the annular parts exhibited another in-plane $\mathrm{B}_{04}$ vibration mode with a spatial phase shift of $\pi / 2$ compared to the aforementioned mode, as shown in Fig. 7(b).

The amplitude-frequency curves were obtained to stimulate the two operating vibration mode under two excitations, as shown in Fig. 7(c). The resonant frequency difference between the two operating vibration modes was $272 \mathrm{~Hz}$, indicating that errors existed during the fabrication and assembly processes of the prototype. Although the frequency differences of corresponding working modes between experimental and calculated results were $1.61 \mathrm{kHz}$ and $1.874 \mathrm{kHz}$, respectively, the measurement results still confirm the validity of our FEM simulation. Errors were caused by neglecting certain calculations during the assembly of the sandwich piezoelectric transducer. The preload forces of the bolts also were difficult to standardize, which resulted in interference modes around the working modes.
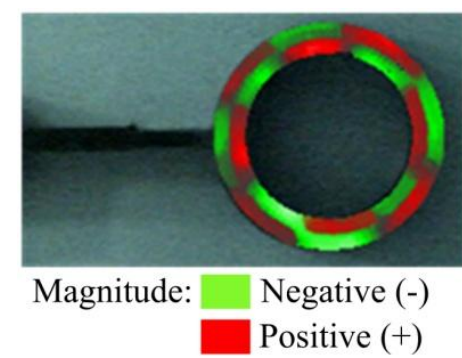

(a)

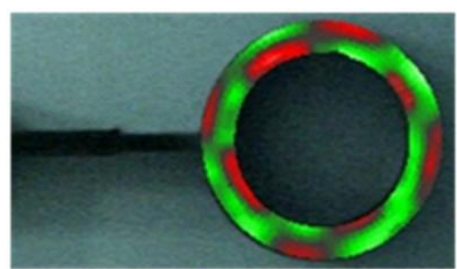

Magnitude: Negative (-)

Positive (+)

(b)

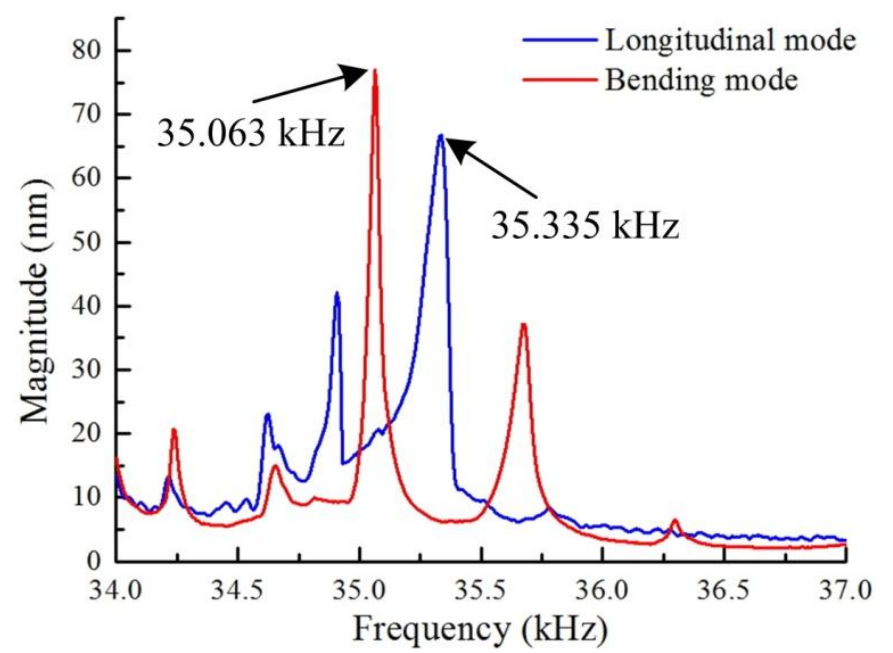

(c)

Fig. 7. Vibration measurement results of the sandwich-type actuating mechanism. Two in-plane $\mathrm{B}_{04}$ vibration modes with a spatial phase difference of $\pi / 2$ were observed in the annular part when the sandwich piezoelectric transducer vibrated at (a) $\mathrm{L}_{3}$ vibration mode and (b) $\mathrm{B}_{10}$ vibration mode, respectively. (c) Amplitude-frequency curves under longitudinal and bending excitations. 


\subsection{Frequency sensitivity}

We successfully measured the resonant frequencies of the operating modes of the actuating mechanism, but were not able to fully determine the driving frequency of the tracked mobile system, because the installation of the metal track changed the boundary of the actuating mechanism and thus influenced the resonant frequencies of the working vibration modes. To identify the optimal driving frequency for the system, we conducted frequency sensitivity experiments.

The motion of the system was recorded by camera throughout the testing process, and then the average speed was obtained after evaluating the videos. We determined the velocity of the system based on the average speed observed during experimentation. A power source used to apply two input electric signals to the tracked mobile system includes two power amplifiers and a two-channel signal generator. An oscilloscope was used to monitor the input signals of the power source including the driven voltages, phases, and frequencies. The tracked mobile system moved onto an aluminum workbench during experimental procedures. Two excitation voltages of $300 \mathrm{~V}_{\mathrm{RMS}}$ with a temporal phase difference of $\pi / 2$ were applied on the sandwich-type actuating mechanism simultaneously. According to the vibration measurement results, we set the driving frequency range from $35 \mathrm{kHz}$ to $36 \mathrm{kHz}$ with a step size of 10 Hz. Results are shown in Fig. 8. The optimal frequency of the system, $35.1 \mathrm{kHz}$, was obtained at maximum velocity of $44.8 \mathrm{~mm} / \mathrm{s}$. As driving frequency increased, the motion speed of the system first increased and then decreased. Compared to the resonant frequency tested in the last section, the driven frequency shifted by $37 \mathrm{~Hz}$, indicating that the installation of the metal track changed the resonant frequency of the operating vibration modes. The optimal driving frequency determined during this experiment was used for all subsequent experiments.

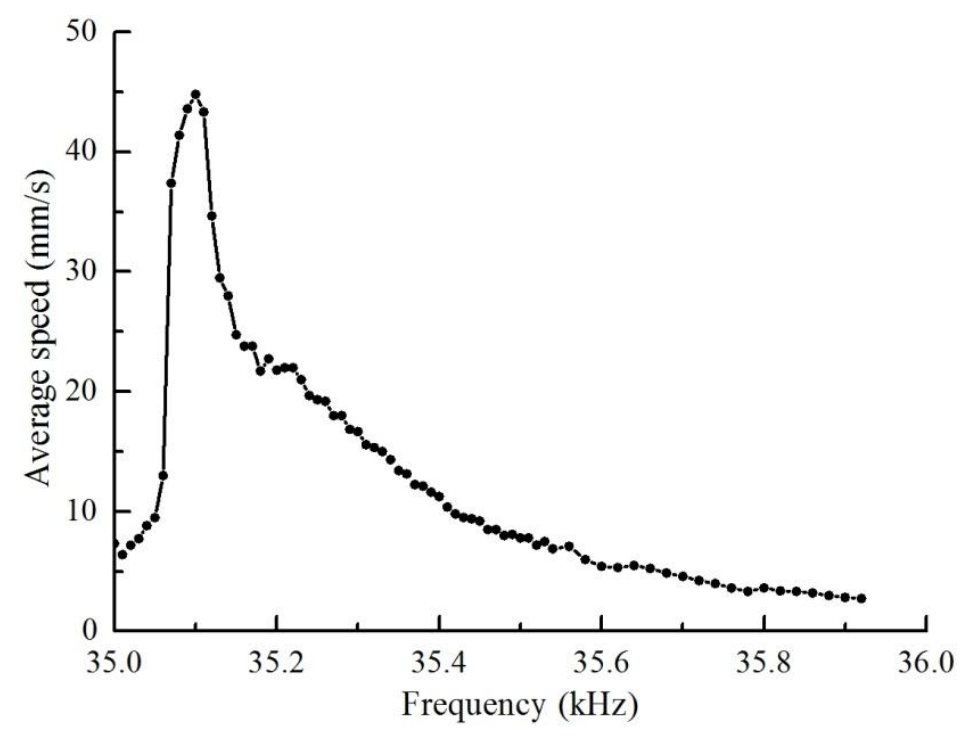

Fig. 8. Frequency sensitivity experiment results.

\subsection{Motion characteristics}

By exchanging the exciting sequence of two applied electric signals, our tracked mobile system can 
achieve bidirectional movement. We tested the dual motion characteristics of the proposed system without load to find that the tracked mobile system prototype still moved onto the aluminum workbench successfully. The power source described above was used to provide two phase electric signals with a temporal phase shift of $\pi / 2$ on the tracked mobile system prototype. The average velocity of the system prototype was also adopted as an evaluation parameter. Figure 9 shows the variations in average velocities of the tracked mobile system prototype under different driving voltages, where the bidirectional motion speeds increased as driving voltage increased; in fact, their relationship was almost linear. It is obviously that the motion performance of the system prototype in the left direction was better than in the right direction at any stimulated voltage; the maximum average velocity in the experimental range was about $72 \mathrm{~mm} / \mathrm{s}$ to the left and $61.5 \mathrm{~mm} / \mathrm{s}$ to the right under the excitation voltage of 500 $\mathrm{V}_{\mathrm{RMS}}$. The reasons for the speed difference on two different sides for the system prototype were: 1) Due to manufacture and fabrication errors, the rotated traveling waves formed in each annular part in both directions were not identical; and 2) the contact interfaces between the actuating mechanism and metal track were not the same on both sides. At driving voltage of $250 \mathrm{~V}_{\mathrm{RMS}}$, the average speeds of the sandwich-type tracked mobile system in left and right directions were $48 \mathrm{~mm} / \mathrm{s}$ and $35 \mathrm{~mm} / \mathrm{s}$, respectively, indicating that its motion speed increased nearly 10 times compared to the previous miniature piezoelectric tracked vehicle [24]. The mobility characteristics of the prototype indeed suggest that the proposed sandwich-type piezoelectric tracked mobile system shows potential application to robotic rover technology in the future.

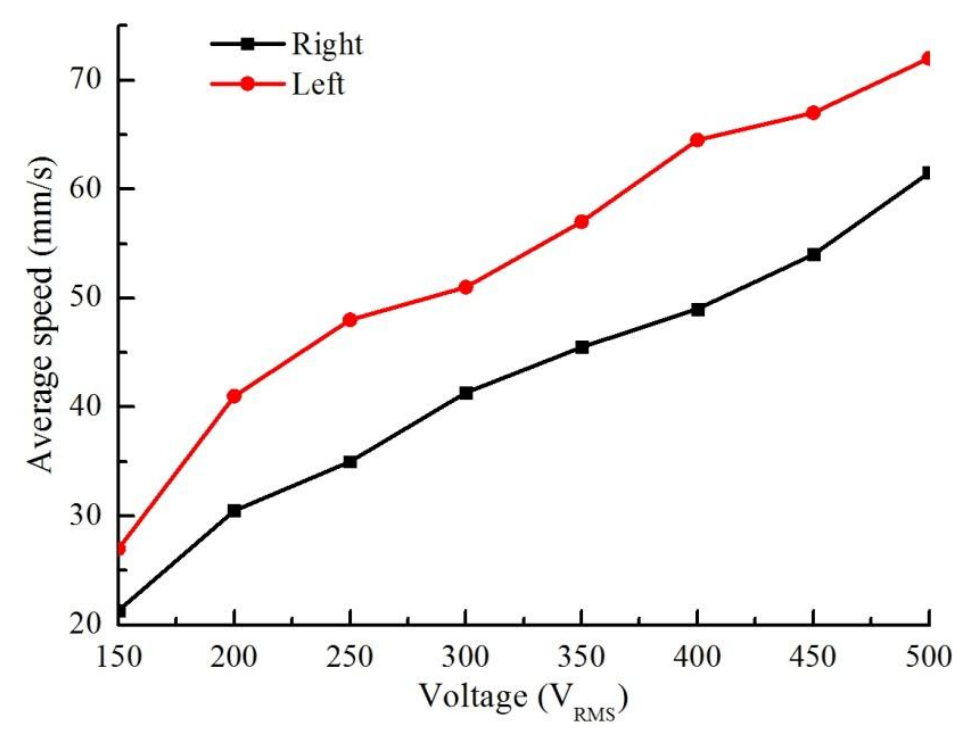

Fig. 9. Bidirectional average speeds of the tracked mobile system prototype versus driving voltages.

\subsection{Loading characteristics}

We built the experimental setup shown in Fig. 10 to measure the loading characteristics of the system prototype, which includes the power source, the oscilloscope, an aluminous platform and a variety of weights. During the experiments, the tracked mobile system moved onto the aluminous platform 
under excitation voltage of $300 \mathrm{~V}_{\mathrm{RMS}}$ while its motion was recorded by camera. Weight was applied incrementally to both sides of the system prototype via a steel rod fixed on the connection block through the conjunct node of the two operating vibration modes of the sandwich piezoelectric transducer. The measured loading characteristics of the prototype during bidirectional movement are shown in Fig. 11, where the maximum speed for the left direction was $59.5 \mathrm{~mm} / \mathrm{s}$ (corresponding to additional weight of $0.32 \mathrm{~kg}$ ) and the maximum speed for the right direction was $61.67 \mathrm{~mm} / \mathrm{s}$ (corresponding to additional weight of $0.24 \mathrm{~kg}$ ), both demonstrating optimal loading state for the tracked mobile system. The maximum additional weight for the left direction was $1.44 \mathrm{~kg}$ and was $1.36 \mathrm{~kg}$ for the right direction when speed was trending to zero, indicating that the ratio of the maximum load to self-weight of the system prototype is nearly 3.57 and 3.37, respectively. The ratios of the maximum load to self-weight of the system prototype basically reflect the inherent mechanical characteristics of the system and state the prospective loading performance. These loading characteristics provide a reference for the future application of this type of tracked mobile system in robotic rovers.

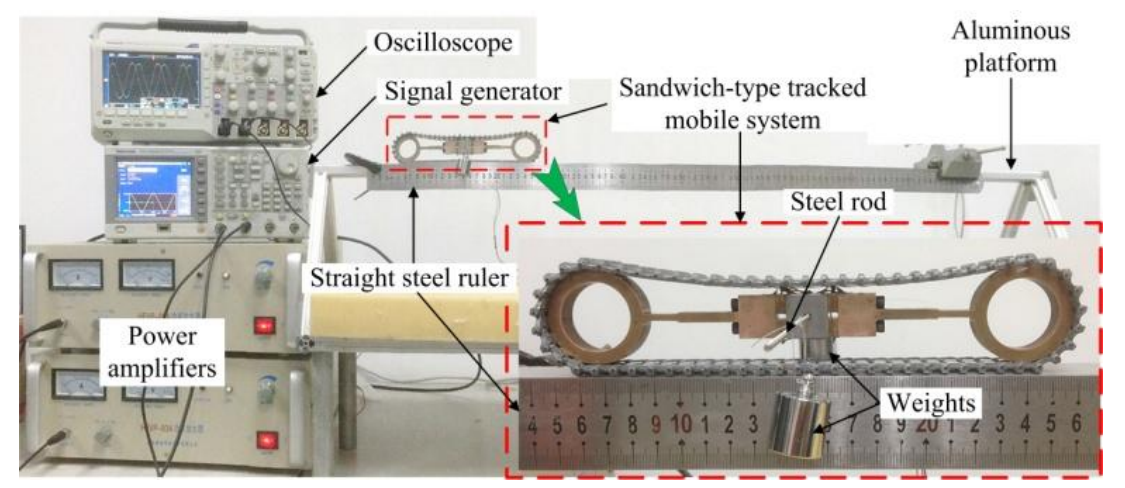

Fig. 10. Experimental setup for measuring the loading characteristics of the sandwich-type tracked mobile system prototype.

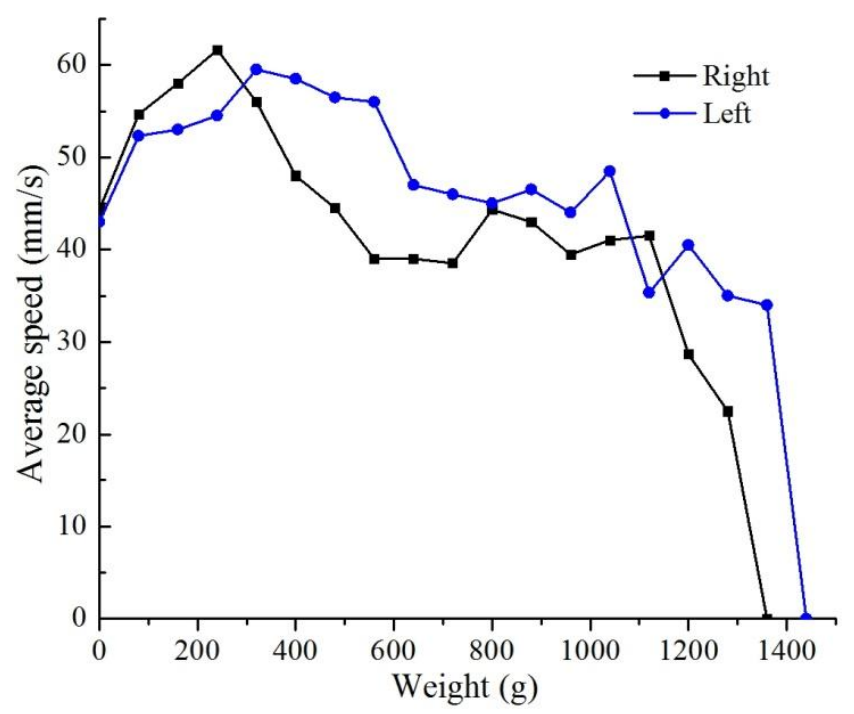

Fig. 11. Loading characteristics of the system prototype.

\section{Conclusions}

A novel sandwich-type traveling wave piezoelectric tracked mobile system was proposed and inves- 
tigated in this study. The tracked mobile system has no sliders or bearings, no bogie wheels, no lubricant, and is actuated by a sandwich-type actuating mechanism, lending it highly reliable and efficient structure that may be well-suited to application in robotic rovers for planetary exploration.

We determined the dimensions of the prototype actuating mechanism based on modal analysis using FEM simulation, then conducted harmonic response analysis to describe the dynamic characteristics of the actuating mechanism, and, finally, manufactured and fabricated a prototype of the tracked mobile system. The measured vibration characteristics of the prototype confirmed the proposed operating principle and FEM calculation results. We also conducted frequency sensitivity experiments to determine the optimal driving frequency of the prototype system to be $35.1 \mathrm{kHz}$, and found that the motion characteristics of the system were very favorable. Its maximum non-load velocity is $72 \mathrm{~mm} / \mathrm{s}$ to the left direction and $61.5 \mathrm{~mm} / \mathrm{s}$ to the right direction corresponding to excitation voltage of $500 \mathrm{~V}_{\mathrm{RMS}}$, and tests with load indicated that the system's ratio of maximum load to self-weight is nearly 3.57 for the left direction and 3.37 for the right direction.

In short, a series of experiments preliminarily demonstrated that the sandwich-type traveling wave piezoelectric tracked mobile system has attractive potential for use in planetary exploration applications. In the future, we plan to investigate the proposed system's ability to cross obstacles and move within extreme environments.

\section{Acknowledgements}

This work was financially supported by the National Science Foundation of China (Grants Nos. 51375225, 51605271 and 91223201), the Project Funded by the Priority Academic Program Development of Jiangsu Higher Education Institutions, and the Postgraduate Research Innovation Projects of Jiangsu Colleges (KYLX16_0332).

\section{References}

[1] C. R. Weisbin, G. Rodriguez, NASA robotics research for planetary surface exploration, IEEE Robot. Automat. Mag., 7 (4) (2000) 25-34. (DOI: 10.1109/100.894030)

[2] P. Harkness, M. Lucas, A. Cardoni, Architectures for ultrasonic planetary sample retrieval tools, Ultrasonics, 51 (8) (2011) 1026-1035. (DOI: 10.1016/j.ultras.2011.06.001)

[3] W.S. Eom, Y.K. Kim, J.H. Lee, G.H. Choi, E.S. Sim, Study on a suspension of a planetary exploration rover to improve driving performance during overcoming obstacles, J. Astron. Space Sci., 29 (4) (2012) 381-387. (DOI: 10.5140/JASS.2012.29.4.381)

[4] E.T. Baumgartner, R.G. Bonitz, J.P. Melko, L.R. Shiraishi, P.C. Leger, A. Trebi-Ollennu, Mobile manipulation for the Mars exploration rover - a dexterous and robust instrument positioning system, IEEE Robot. Automat. Mag., 13 (2) (2006) 27-36. (DOI: 10.1109/MRA.2006.1638013)

[5] C.A. Brooks, K. Iagnemma, Vibration-based terrain classification for planetary exploration rovers, IEEE Trans. Robot., 21 (6) (2005) 1185-1191. (DOI: 10.1109/TRO.2005.855994) 
[6] J. Morales, J.L. Martinez, A. Mandow, A.J. Garcia-Cerezo, S. Pedraza, Power consumption modeling of skid-steer tracked mobile robots on rigid terrain, IEEE Trans. Robot., 25 (5) (2009) 1098-1108. (DOI: 10.1109/TRO.2009.2026499)

[7] J.G. Marquardt, J. Alvarez, K.D. von Ellenrieder, Characterization and system identification of an unmanned amphibious tracked vehicle, IEEE J. Ocean. Eng., 39 (4) (2014) 641-661. (DOI: 10.1109/JOE.2013.2280074)

[8] Z. Luo, J. Shang, Z. Zhang, A reconfigurable tracked mobile robot based on four-linkage mechanism, J. Cent. South Univ., 20 (1) (2013) 62-70. (DOI: 10.1007/s11771-013-1460-8)

[9] Y. Liu and G. Liu, Track-stair interaction analysis and online tipover prediction for a self-reconfigurable tracked mobile robot climbing stairs, IEEE/ASME Trans. Mechatronics, 14(5) (2015) 528-538. (DOI: 10.1109/TMECH.2009.2005635)

[10] W. Rao, J.D. Shi, J.Z. Wang, Dynamic analysis for articulated-tracked robot climbing stairs, Advanced Materials Research, 889-890 (2014) 483-487. (DOI: 10.4028/www.scientific.net/AMR.889-890.483)

[11] B. Wu, Z.J. Wei, Twin-tracked robot obstacle three-dimensional modeling and simulation, Advanced Materials Research, 479-481 (2012) 2351-2354. (DOI: 10.4028/www.scientific.net/AMR.479-481.2351)

[12] Y. Liu, G. Liu, Interaction analysis and online tip-over avoidance for a reconfigurable tracked mobile modular manipulator negotiating slopes, IEEE/ASME Trans. Mechatronics, 15 (4) (2010) 623-635. (DOI: 10.1109/TMECH.2009.2031174)

[13] K.W. Wait, M. Goldfarb, A pneumatically actuated quadrupedal walking robot, IEEE/ASME Trans. Mechatronics, 19 (1) (2014) 339-347. (DOI: 10.1109/TMECH.2012.2235078)

[14] Y. Liu, G. Liu, Modeling of tracked mobile manipulators with consideration of track-terrain and vehicle-manipulator interactions, Robot. Auton. Syst., 57 (11) (2009) 1065-1074. (DOI: 10.1016/j.robot.2009.07.007)

[15] X. Gao, K. Li, J. Gao, A mobile robot platform with double angle-changeable tracks, Adv. Robot., 23 (9) (2009) 1085-1102. (DOI: $10.1163 / 156855309 X 452548)$

[16] A. Ellery, Environment-robot interaction-the basis for mobility in planetary micro-rovers, Rob. Autom. Syst., 51 (1) (2005) 29-39. (DOI: 10.1016/j.robot.2004.08.007)

[17] A. Schiele, J. Romstedt, C. Lee, H. Henkel, S. Klinkner, R. Bertrand, R. Rieder, R. Gellert, G. Klingelhofer, B. Bernhardt, H. Michaelis, Nanokhod exploration rover: A rugged rover suited for small, low-cost, planetary lander mission, IEEE Robot. Automat. Mag., 15 (2) (2008) 96-107. (DOI: 10.1109/MRA.2008.917888)

[18] I. Rekleitis, J.L. Bedwani, E. Dupuis, Over-the-horizon, autonomous navigation for planetary exploration, in Proc. IEEE/RSJ Int. Conf. Int. Robots and Systems, (2007) 2248-2255. (DOI: 10.1109/IROS.2007.4399533)

[19] M. Li, B. Huang, G. Liu, L. Sun, A modular reconfigurable tracked micro-robot, Robot, 28 (5) (2006) 548-552. (DOI: http://robot.sia.cn/EN/Y2006/V28/I5/548)

[20] Y. Shi, Y. Li, C. Zhao, J. Zhang, A new type butterfly-Shaped transducer linear ultrasonic motor, J. Intelligent Material Syst. Struct. 22 (2011) 567-575. (DOI: $\underline{\text { 10.1177/1045389X11404955) }}$

[21] Y. Liu, W. Chen, J. Liu, S. Shi, A cylindrical standing wave ultrasonic motor using bending vibration transducer, Ultrasonics, 51 (5) (2011) 527-531. (DOI: 10.1016/j.ultras.2010.12.007)

[22] Z.J. Chen, X.T. Li, P.H. Ci, G.X. Liu, S.X. Dong, A standing wave linear ultrasonic motor operating in in-plane expanding and bending modes, Rev. Sci. Instrum., 86 (3) (2015), p. 035002. (DOI: $\underline{10.1063 / 1.4914843}$ )

[23] Y. Liu, D. Xu, X. Yang, W. Chen, Miniaturized piezoelectric actuator operating in bending hybrid modes, Sens. Actuators A, 235 (2015) 158-164. (DOI: 10.1016/j.sna.2015.10.002)

[24] X. Yang, Y. Liu, W. Chen, J. Liu, A cylindrical traveling wave ultrasonic motor using bonded-type composite beam, U1trasonics, 65 (2016) 277-281. (DOI: 10.1016/j.ultras.2015.09.014)

[25] Z. Dong, M. Yang, Z. Chen, L. Xu, F. Meng, W. Ou, Design and performance analysis of a rotary traveling wave ultrasonic motor with double vibrators, Ultrasonics, 71 (2016) 134-141. (DOI: 10.1016/j.ultras.2016.06.004)

[26] X. Li, Z. Yao, S. Zhou, Q. Lv, Z. Liu, Dynamic modeling and characteristics analysis of a modal-independent linear, Ultrasonics, 72 (2016) 117-127. (DOI: 10.1016/j.ultras.2016.07.018) 
[27] J. Jin, F. Qian, Y. Yang, J. Zhang, K. Zhu, A piezoelectric tracked vehicle with potential application to planetary exploration. Chin. Sci. Bull., 57 (11) (2012) 1339-1342. (DOI: 10.1007/s11434-012-5004-7)

[28] C. Huang, L. Wang, C. Shu, H. Zhang, J. Jin, Contact friction behavior of piezoelectric vibrator for tracked vehicle driving, J. Vibration, Measurement and Diagnosis, 35 (1) (2015) 170-177. (in Chinese) (DOI: 10.16450/j.cnki.issn. 1004-6801.2015.01.029) 